\title{
Hepatocyte Growth Factor Prevents Renal Fibrosis and Dysfunction in a Mouse Model of Chronic Renal Disease
}

\author{
Shinya Mizuno, ${ }^{\star \ddagger}$ Tsutomu Kurosawa, ${ }^{*}$ Kunio Matsumoto, ${ }^{\ddagger}$ Yoko Mizuno-Horikawa, ${ }^{*}$ Munehiro Okamoto, ${ }^{\star}$ \\ and Toshikazu Nakamura‡ \\ *The Institute of Experimental Animal Sciences, and ${ }^{\ddagger}$ Division of Biochemistry, Department of Oncology, Biomedical Research Center, \\ Osaka University Medical School, Suita 565-0871, Japan
}

\begin{abstract}
Chronic renal disease (CRD) is generally thought to be incurable, except through renal transplantation, and the number of patients with CRD is on the increase. Glomerulosclerosis and tubulointerstitial fibrosis represent the morphological equivalent of end-stage CRD. In this study, we demonstrated the preventive effect of hepatocyte growth factor (HGF) on the progression of renal dysfunction and fibrosis, using a spontaneous mouse model for CRD (ICGN strain). The mice progressively developed glomerular sclerotic injury, tubular atrophy, and renal dysfunction until they were 17 wk of age. When recombinant HGF was injected into these mice during a 4-wk-period (from weeks 14-17 after birth), DNA synthesis of tubular epithelial cells was found to be 4.4-fold higher than in mice without HGF injection, thereby suggesting tubular parenchymal expansion promoted by HGF. Notably, HGF suppressed the expression of transforming growth factor- $\beta$ and of platelet-derived growth factor as well as myofibroblast formation in the affected kidney. Consequently, the onset of tubulointerstitial fibrosis was almost completely inhibited by HGF, while HGF attenuated the progression of glomerulosclerosis, both leading to preventing manifestation of renal dysfunction. From our results, supplement therapy with HGF may be taken into consideration as a novel option for prevention and treatment of CRD. (J. Clin. Invest. 1998. 101:18271834.) Key words: chronic renal failure - HGF therapy • glomerulosclerosis $\bullet$ kidney $\bullet$ tubular proliferation
\end{abstract}

\section{Introduction}

Dialysis market estimates indicate that $>400,000$ patients world-wide have undergone maintenance dialysis, and the number of patients with chronic renal disease $(\mathrm{CRD})^{1}$ is on the increase. The major initial etiology of CRD is chronic glomerulonephritis and diabetic nephropathy (1). Glomerulosclerosis and tubulointerstitial fibrosis, characterized by a progressive

Address correspondence to Dr. Tsutomu Kurosawa, The Institute of Experimental Animal Sciences, Osaka University Medical School, Yamadaoka 2-2, Suita 565-0871, Japan. Phone: 81-6-879-3171; FAX: 81-6-879-3107; E-mail: kurosawa@iexas.med.osaka-u.ac.jp

Received for publication 11 September 1997 and accepted in revised form 5 February 1998.

J. Clin. Invest.

(C) The American Society for Clinical Investigation, Inc. 0021-9738/98/05/1827/08 \$2.00

Volume 101, Number 9, May 1998, 1827-1834

http://www.jci.org loss of parenchymal cells and overdeposition of extracellular matrix (ECM), represent the morphological equivalent of endstage CRD and occur independently of the primary underlying disorder. Thus, suppression of the shift to the common final pathway appears to be a reasonable strategy for the treatment of CRD.

With regard to the pathogenesis of renal fibrosis, glomerular injury has been thought to play an important role in progression to renal failure, even if it is still unclear whether injured glomeruli cause or reflect the fibrotic disease $(2,3)$. In contrast, there is now ample histopathological evidence showing that tubulointerstitial fibrosis is the most important predictor for prognosis of various primary and secondary glomerular diseases of diverse etiology $(4,5)$. In most patients with glomerular diseases, the decline in renal function correlates more closely with the extent of tubular and tubulointerstitial lesions than with that of glomerular injury. Therefore, much interest has now begun to center on tubular and tubulointerstitial compartments $(3,6-8)$.

It is well known that compensatory growth of tubular components occurs, in the injured or resected renal tissues, in a fashion of epithelial cellular hypertrophy and hyperplasia, to protect the renal organ against a loss of functional nephrons (9). Indeed, renal function is well-preserved as long as $>20 \%$ of the portion in nephrons remains intact. These backgrounds led us to hypothesize that expansion of tubular parenchymal components in intact remnant nephron could minimize the onset of tubulointerstitial fibrosis and prohibit clinical manifestation of renal failure. On the basis of this current notion, we directed attempts to induce tubular compensatory growth and to prevent the onset of tubulointerstitial fibrosis by administering a biomodulator, such as a cytokine or a growth factor with potent tubulogenetic activity.

Hepatocyte growth factor (HGF) was originally identified and cloned as a potent mitogen for mature hepatocytes (10, 11). HGF induces mitogenic and morphogenic responses in renal tubular cells $(12,13)$. Expression of the HGF or its receptor, c-met, is rapidly upregulated in remnant kidney of nephrectomized rats as a trigger for compensatory growth $(14,15)$. Furthermore, HGF was shown recently to accelerate regeneration of tubular parenchymal components after acute renal injury in mice $(16,17)$. Therefore, HGF is considered to be a candidate for a renotrophic factor(s). Thus, we attempted to prevent the tubulointerstitial fibrosis with this renotrophic factor.

1. Abbreviations used in this paper: $\mathrm{ABC}$, avidin-biotin coupling; BrdU, 5-bromo-2'-deoxyuridine; BUN, blood urea nitrogen; CRD, chronic renal disease; ECM, extracellular matrix; HGF, hepatocyte growth factor; PAS, periodic acid-Schiff; sAlb, serum albumin; $\mathrm{sCr}$, serum creatinine; $\alpha$-SMA, $\alpha$-smooth muscle actin; uAlb, urinary albumin. 
The nephrotic mouse (ICGN strain) is a unique model for naturally occurring nephrotic syndrome (18-20). The mice exhibit glomerular injury and heavy proteinuria, and then finally develop renal failure. Recently, we found that the nephrotic mice manifest renal dysfunction in parallel with the progression of the tubular rather than glomerular injury (20). In the present study, we used the nephrotic mice as an experimental analogue of human CRD and found that administration of HGF, the potent renotrophic factor, did elicit a therapeutic effect by enhancing proliferation of tubular epithelial cells and inhibiting renal fibrosis.

\section{Methods}

Recombinant human HGF. A variant type of human recombinant HGF was produced by Chinese hamster ovary cells, a cell line transfected with human HGF cDNA with a deletion of 5 amino acid residues in the first kringle domain. This variant HGF has the same biological activities as native HGF, but is slightly more potent in a specific condition (21). Purity of the HGF protein was confirmed to be $>98 \%$.

Animals and HGF injections. The nephrotic mice, as determined by albuminuria (20), were used in this study. The mice were identified to be in an early stage of renal insufficiency when their blood urea nitrogen (BUN) levels reached $\sim 40 \mathrm{mg} / \mathrm{dl}$, and then were divided into an HGF-injected group and a saline-injected control group. No significant difference in laboratory data before the experiment was seen between these two groups. In the HGF-injected group, mice (three male and three female) were administered subcutaneously daily with $500 \mu \mathrm{g} / \mathrm{kg}$ HGF for $28 \mathrm{~d}$, whereas mice (three male and three female) in the control group received subcutaneous injections of an identical volume of saline.

Serum and urine chemistry. Serum was obtained on days $0,8,18$, and 28. Urine, accumulated for a 12-h period, was stocked on the same time point. The urine and serum samples were subjected to the following tests (20). The BUN level was measured by a urease indophenol method (urea nitrogen-B test; Wako, Osaka, Japan). The serum creatinine ( $\mathrm{sCr}$ ) level was measured by Jeff's method (creatinine test; Wako). The urinary albumin (uAlb) and serum albumin (sAlb) levels were determined by a bromcresol green method (A/G B test; Wako).

Evaluation of tubular injury, and immunohistochemical analyses for tubular proliferation and interstitial ECM deposition. At the scheduled necropsy, the kidney was removed, fixed in neutral buffered formalin ( $\mathrm{pH} 7.4$ ), and embedded in paraffin. Each section was cut into 4- $\mu \mathrm{m}$ slices, stained with periodic acid-Schiff (PAS) reagent and observed under a light microscope. Tubular injury, characterized by the tubular dilatation, epithelial cellular atrophy, and luminal cast formation with tubulointerstitial expansions, was graded according to the extent of cortical involvement on a scale of 0 to 4 : 0 , normal; 0.5 , small focal area of the tubular injury; 1 , involvement of $>10 \%$ of the cortex; 2 , involvement of up to $25 \%$ of the cortex; 3 , involvement of up to $50-75 \%$ of the cortex; and 4 , extensive damage involving $>75 \%$ of the cortex $(7,20)$. To evaluate in vivo mitogenic activity of HGF, renal cells undergoing DNA synthesis were examined on the HGFinjected mice and control mice by 5-bromo-2'-deoxyuridine (BrdU) incorporation into DNA and immunohistochemical staining (16). The mice were intraperitoneally injected with BrdU (Sigma, St. Louis, $\mathrm{MO}$ ) at a dose of $100 \mathrm{mg} / \mathrm{kg}$ and were killed $1 \mathrm{~h}$ after the BrdU injection. To assay the BrdU labeling, the tissues were fixed with $70 \%$ ethanol at $4^{\circ} \mathrm{C}$ for $12 \mathrm{~h}$, dehydrated, and embedded in paraffin. Sections were subjected to immunohistochemistry for BrdU labeling (16), and the BrdU labeling index was determined by counting $>1,000$ nuclei in randomly selected microscopic fields. To detect the ECM proteins, the remaining kidney tissues were fixed in the neutral buffered formalin and paraffin-embedded. An avidin-biotin coupling (ABC) im- munoperoxidase technique was performed on the dewaxed sections, using a commercial kit (Vectastain Elite $\mathrm{ABC}^{\mathrm{TM}}$; Vector Laboratories, Burlingame, CA), according to the instructions of the manufacturer. Antibodies used for the primary reactions were rabbit $\mathrm{IgG}$ against rat type I collagen (1:200) (Cosmo-Bio, Tokyo, Japan) and rabbit IgG against mouse fibronectin (1:400) (Chemicon International, Temecula, CA). The immunostainings for type I collagen and fibronectin were semiquantified by morphometric analysis, using a microvideoscope system (VM-30; Olympus, Tokyo, Japan), and were expressed as a percentage of tubulointerstitial space labeled with the antibody relative to total tubular area (labeled area/total area $\times 100$ ).

Immunohistochemical analyses of the antifibrotic effect by $H G F$. Fibrogenetic growth factors such as PDGF and TGF- $\beta$ may be involved in the myofibroblast formation and the progression of tubulointerstitial fibrosis $(6,7)$. All the renal tissues were fixed in neutral buffered formalin, except for fixation with $70 \%$ ethanol to detect TGF- $\beta$ and embedded in paraffin. To visualize expressions of the growth factors, goat IgG against PDGF (1:100) (Becton Dickinson, Bedford, MA) and rabbit IgG against TGF- $\beta$ (1:30) (R\&D Systems, Minneapolis, MN) were applied on the dewaxed sections for the primary reactions, followed by the $\mathrm{ABC}$ immunoperoxidase technique. To identify myofibroblasts, a direct technique using a kit of peroxidase-conjugated mouse monoclonal $\operatorname{IgG}$ against human $\alpha$-smooth muscle actin ( $\alpha$-SMA) (DAKO, Glostrup, Denmark) was used. The PDGF expression was semiquantified as a percentage of distal tubules labeled with the antibody against PDGF relative to total distal tubule. For immunostaining for the $\alpha$-SMA or TGF- $\beta$ proteins, at least 20 randomly chosen nonoverlapping high-power fields $(\times 400$ magnification) were examined, and the mean number of $\alpha$-SMA-positive cells or TGF- $\beta$-positive cells per high-power field was determined in a blind fashion.

Morphometric and immunohistochemical analyses of glomerular lesions. The effect of HGF on glomerulosclerosis was also investigated on the same sections (PAS stain) as the tubular histopathology. The glomerular matrix score was determined by a well-established method, based on the extension of mesangial expansions (22). The intraglomerular cell number was counted on the same glomeruli as measured for the matrix score. To further clarify the effects on the glomerular sclerogenetic events, TGF- $\beta$-positive cells per glomerulus were counted on the same sections as those used for the immunohistochemical analyses of tubulointerstitial fibrosis. To evaluate the myofibroblast formation and ECM deposition, expressions of $\alpha$-SMA and type I collagen were examined on at least 30 glomeruli, and degrees of the expressions were semiquantified with immunostaining scores, as defined by Johnson and co-workers $(7,23)$.

Measurement of TGF- $\beta$ and PDGF proteins by ELISA. Renal tissue segments were homogenized in $10 \mathrm{mM}$ Tris- $\mathrm{HCl}$ buffered solution ( $\mathrm{pH}$ 7.4) containing $2 \mathrm{M} \mathrm{NaCl}, 1 \mathrm{mM}$ PMSF, 1 mM EDTA, and $0.01 \%$ Tween 80 , and was centrifuged at $15,000 \mathrm{rpm}$ for $30 \mathrm{~min}$. For measurements of TGF- $\beta$ and PDGF proteins, the tissue extracts were applied to a 96-well microplate in commercial ELISA kits (Quantikine kit ${ }^{\mathrm{TM}} ; \mathrm{R} \& \mathrm{D}$ Systems). The colorimetric measurements were performed according to the manufacturer's instructions.

Statistical analyses. All data were expressed as mean $\pm \operatorname{SE}(n=6)$. A Student's $t$ test or ANOVA test for parametric values and MannWhitney U test for nonparametric values were used to compare group means, with $P<0.05$ accepted as significant.

\section{Results}

Therapeutic effects of HGF on clinical symptoms. The control CRD mice progressively manifested clinical signs of depression, appetite, pale ears, body weight loss, and occasional edema, while the HGF-injected mice showed no signs and remained active throughout the HGF injections. Renal dysfunction was evaluated on the basis of the BUN and sCr levels (Fig. $1 \mathrm{~A}$ ). The BUN level in the control mice progressively in- 

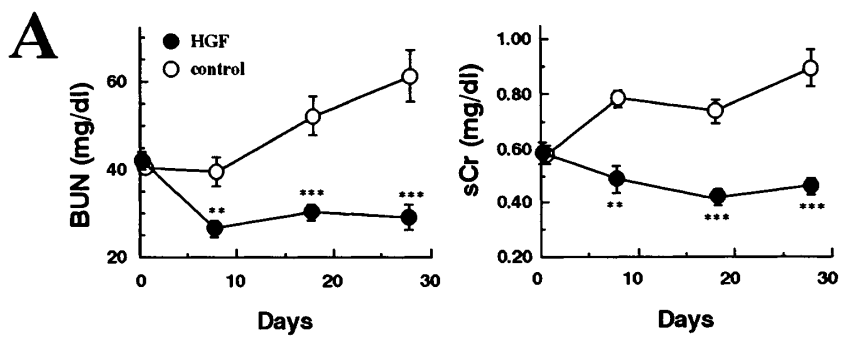

B
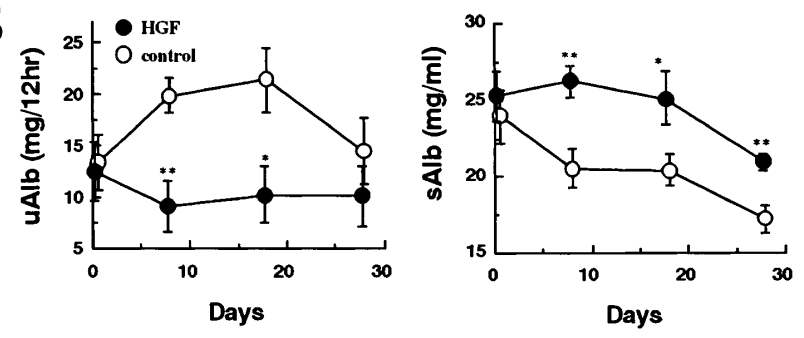

Figure 1. Therapeutic effects of HGF injections on progression of CRD. ( $A$ ) Inhibitory effect of HGF on progression of renal dysfunction, as determined by time courses of BUN and sCr levels. $(B)$ Delaying effect of HGF on the development of nephrotic syndrome, as determined by changes in the uAlb excretion and sAlb levels. Vertical bar represents SEM $(n=6)$. Statistical analysis: $* P<0.05$, ** $P<$ 0.01 , and $* * * P<0.001$ compared with the same time point of the control group.

creased from $40.6 \pm 1.2 \mathrm{mg} / \mathrm{dl}$ on day 0 to $61.2 \pm 5.8 \mathrm{mg} / \mathrm{dl}$ on day 28, while BUN levels in the HGF-injected mice decreased from $42.1 \pm 2.2 \mathrm{mg} / \mathrm{dl}$ on day 0 to $26.5 \pm 1.9 \mathrm{mg} / \mathrm{dl}$ on day 8 and this reduced level was still maintained by day 28 . Consistent with the decrease in BUN levels, sCr levels in the HGF-injected mice also reverted to the normal range, and $\mathrm{sCr}$ levels of the HGF-injected mice on day 28 decreased to $52 \%$ of the control value. The nephrotic states were evaluated on the basis of the albuminuria and hypoalbuminemia levels (Fig. $1 B$ ). An $\sim 1.6-$ fold increase in the uAlb excretion, noted on days 8-18 in the control mice, was diminished by HGF injections. sAlb levels in the control mice decreased gradually throughout the experiment, while those in the HGF-injected mice remained unchanged, except for a decrease on day 28. We have no evidence to explain the reduction in albuminuria, but recent work has demonstrated mitogenic activity of HGF in glomerular epithelial cells (24). In addition, since injury to the glomerular epithelial cells is considered to be one of the causes of albuminuria (25), HGF may attenuate progressive albuminuria through preventing the glomerular epithelial injury. Further analyses are needed to clarify the antialbuminuric mechanism. Explanations for the improvement in hypoalbuminemia include not only the attenuated albuminuria but also possible hepatic albumin synthesis by HGF (26).

Tubular proliferation and reduced tubulointerstitial fibrosis by HGF injections. Tubular atrophy and intraluminal cast accumulation with tubulointerstitial expansion were severely and extensively observed in the cortical area of the control mice (Fig. $2 A$ ). In contrast, the tubular lesion was mild and the tubular structures were well-preserved within the kidneys of the HGF-treated mice. The tubular injury score in the HGFtreated mice was reduced to $25 \%$ of that of the control mice (Fig. $2 E$ ). In the control kidney, there were few tubular cells positive for the BrdU labeling (Fig. 2 B). In contrast, tubular cells undergoing DNA synthesis were observed in both proximal and distal tubules of the mice treated with HGF. The labeling indices for mice given saline alone and HGF were $1.16 \pm 0.08$ and $5.13 \pm 0.53 \%(P<0.001)$, respectively (Fig. 2 $E)$. Degrees of the deposited ECM proteins were examined to confirm the antifibrotic effect of HGF. Deposition of type I collagen in the interstitial spaces was found severe in the control mice and mild in the HGF-injected mice (Fig. $2 C$ ). The field labeled with type I collagen in the interstitial spaces of HGF-treated mice was reduced to $13 \%$ of the control level (Fig. $2 E$ ). In addition, tubulointerstitial deposition of fibronectin was less in the HGF-injected than in the control mice (Fig. $2 D$ ). The tubulointerstitial field labeled with fibronectin of the HGF-injected mice accounted for only $28 \%$ of that of the control mice (Fig. $2 E$ ). These findings support our hypothesis that HGF-induced tubular growth minimizes the tubulointerstitial fibrosis. HGF seems to prohibit the expansion of interstitial space through proliferating the parenchymal cellular components, as reported in a liver cirrhosis model (27).

Inhibitory effects on molecular and cellular events leading to tubulointerstitial fibrosis. The possible etiological roles of PDGF and TGF- $\beta$ in numerous disease processes, in particular the chronic inflammatory fibrotic disorder, have been well recognized $(6,7,28,29)$. Therefore, we analyzed the expression of TGF- $\beta$ and PDGF in the kidney of the CRD mice. The PDGF protein was predominantly expressed in the distal tubules of the control mice, while in general, few distal tubules positive for PDGF were seen in mice given HGF (Fig. $3 A$ ). The percentage of distal tubules expressing PDGF in the HGF-injected mice was significantly lower than in mice given saline alone $(12.5 \pm 1.93$ vs. $47.2 \pm 5.54 \%, P<0.001)$ (Fig. $3 \mathrm{D})$. In addition, positive immunolabeling for TGF- $\beta$ was detected in macrophages or fibroblast-like cells around areas of active fibrosis in the control mice (Fig. $3 \mathrm{~B}$ ). In the HGF-treated mice, positive immunolabeling for the TGF- $\beta$ protein was uncommon, accompanied by a significant decrease of TGF- $\beta$-positive cells compared with the control mice (Fig. $3 D$ ). In the control mice, myofibroblasts, identified as spindle-shaped cells with positive labeling for $\alpha$-SMA, were observed in the tubulointerstitial spaces at the cortical and corticomedullar and papillary areas (Fig. $3 C$ ). In contrast, myofibroblast formation in the HGFtreated mice was reduced to only $10 \%$ of the control level (Fig. $3 D$ ). In addition, the expression of PDGF in the proximal tubules was found weak but extensive in the HGF-treated mice but negligible in the control mice (data not shown). Although the roles of PDGF in the pathogenesis of tubulointerstitial fibrosis are unknown, PDGF expressed in the lower tubules may be directly or indirectly involved in the evolution of tubulointerstitial fibrosis, as observed previously (30). With regard to this, an in vitro study suggests that PDGF released from a lower tubular cell line could promote the fibroblast proliferation and cause tubulointerstitial fibrosis (31). HGF may attenuate the fibrogenesis by reducing the PDGF expression.

Delaying effects of HGF on progression of glomerulosclerosis. Glomerulosclerosis, characterized by the mesangial expansion and cellular atrophy, was severely noted in the control mice, whereas the glomerular lesion was moderate in the HGF-treated mice (Fig. $4 A$ ). The glomerular matrix score for the HGF-treated mice was reduced to $65 \%$ of that for the control mice (Fig. $4 E$ ). In addition, loss of the glomerular paren- 

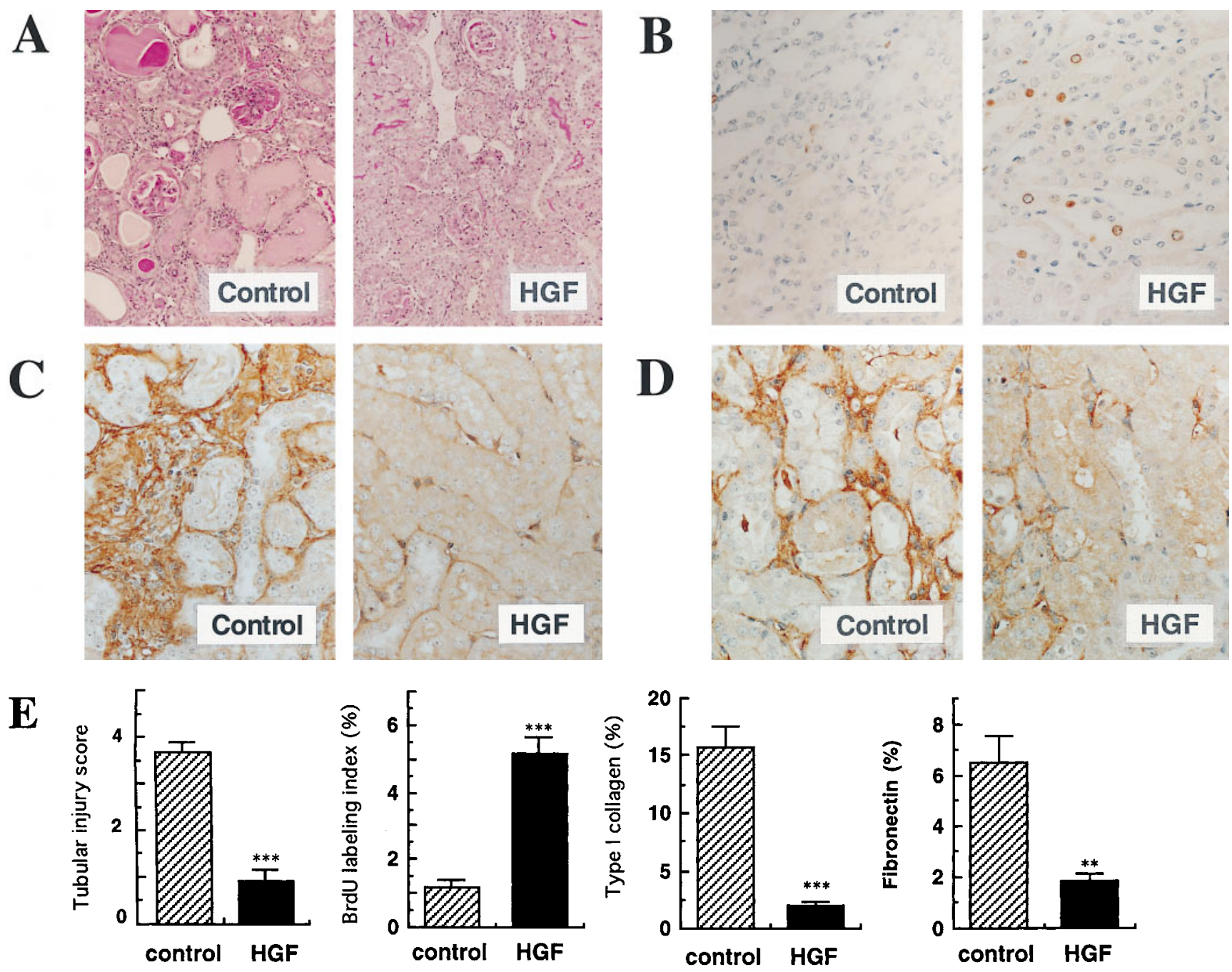

Figure 2. Beneficial effects of HGF injections on tubular injury and tubulointerstitial fibrosis. $(A)$ Representative photomicrographs of tubular lesions $(\times 60$, PAS stain). $(B)$ Detection of tubular cell proliferation by BrdU incorporation $(\times 160)$. $(C$ and $D)$ Tubulointerstitial fibrosis, as determined by depositions of type I collagen $(C)$ and fibronectin $(D)(\times 160)$. $(E)$ Semiquantification of these histological findings $(m e a n \pm$ SE, $n=$ 6). Statistical analysis: $* * P<0.01$ and $* * * P<0.001$ compared with the control group.

chymal cells was significantly suppressed in the HGF-injected mice compared with the control mice (cell count per glomerulus: $29.9 \pm 0.89$ vs. $26.0 \pm 0.98, P<0.01$, data not illustrated). Interestingly, TGF- $\beta$-positive cells in the glomeruli were significantly reduced in the HGF-injected mice compared with the control mice (Fig. 4, $B$ and $E$ ). Furthermore, periglomerular myofibroblast formation, as evaluated by $\alpha$-SMA expression, was markedly suppressed in the HGF-injected mice (Fig. 4, C and $E$ ). Deposition of type I collagen, a specific ECM protein for interstitial area, was severely noted in the glomeruli of the control mice (Fig. $4 D$ ). In contrast, the score of the ECM protein was significantly lower in the HGF-treated mice than in the control mice (Fig. $4 E$ ).

$T G F-\beta$ and PDGF levels in renal tissues. When renal TGF- $\beta$ levels were determined by ELISA, TGF- $\beta$ levels in the control and HGF-treated mice were $1666.7 \pm 256.2$ and $275.7 \pm 22.2 \mathrm{ng} / \mathrm{g}$ tissue, respectively. Renal TGF- $\beta$ concentration in the HGFtreated CRD mice was decreased to $17 \%$ of that of the untreated mice (Fig. 5 A). Similarly, the renal PDGF level in the
HGF-treated mice was decreased to $28 \%$ of that of the control mice (Fig. $5 \mathrm{~B}$ ). These findings were consistent with the immunohistochemical findings, as described above.

\section{Discussion}

In contrast to what its name suggests, HGF targets several epithelial cells in multiple organs (32). The in vivo effect of HGF injections on affected organs was initially studied by using animal models of acute hepatic injury (33) and acute renal failure $(16,17)$. With regard to chronic diseases, HGF was demonstrated recently to be effective in retarding progression of liver cirrhosis $(27,34,35)$ or pulmonary fibrosis (36). In the present study, we found that HGF inhibited the progression of renal fibrosis and dysfunction in a murine model on course for chronic renal failure. To our knowledge, this is the first report demonstrating the potential of growth factors to inhibit clinical manifestations of CRD. 

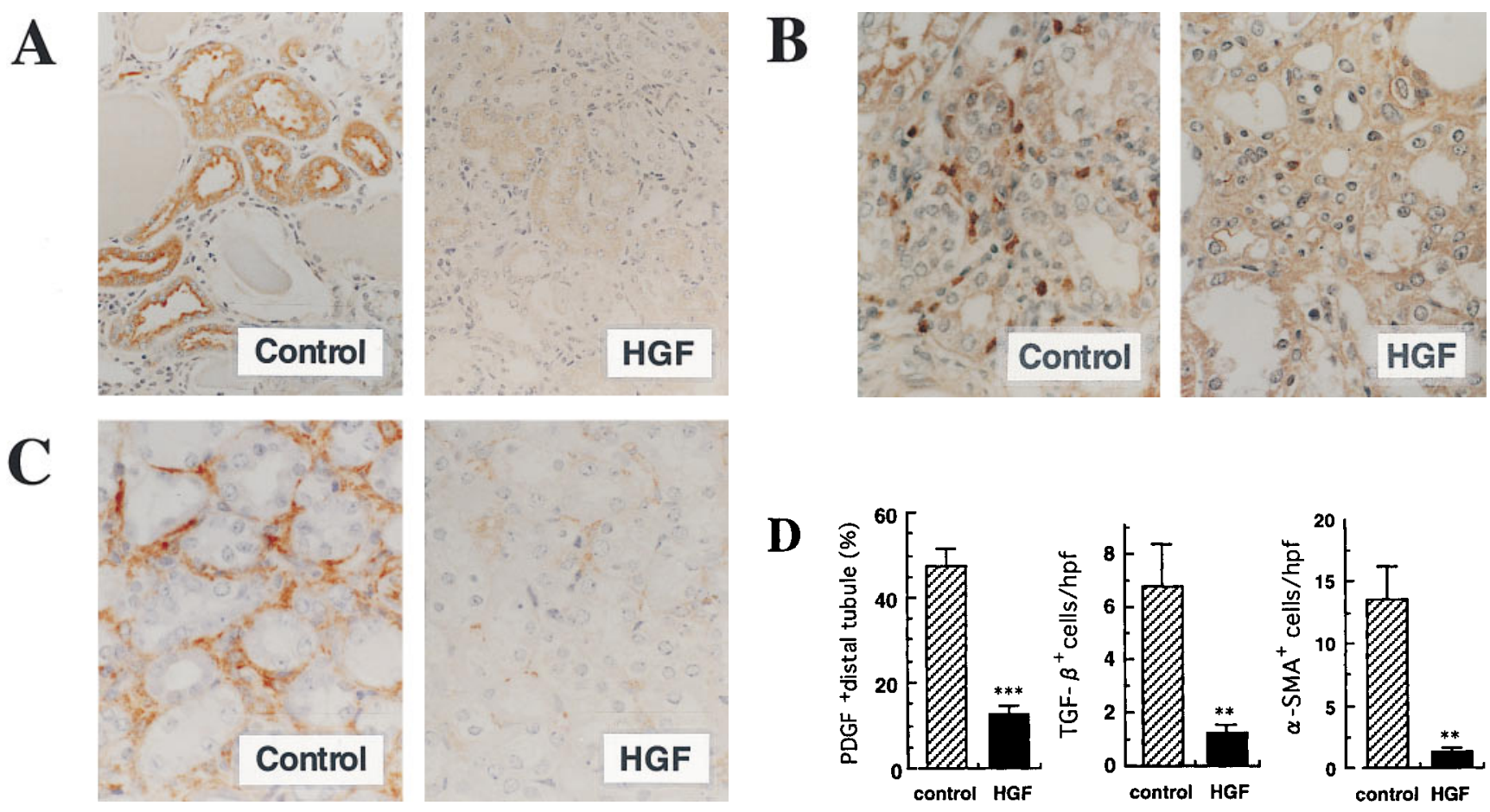

Figure 3. Inhibition of fibrogenetic events in tubular and tubulointerstitial areas by HGF injections. $(A)$ Immunostaining for PDGF localized in distal tubules $(\times 130)$. (B) Immunostaining for TGF- $\beta$ localized in macrophage- or fibroblast-like cells $(\times 220)$. $(C)$ Immunostaining for myofibroblasts, as identified as $\alpha$-SMA-positive cells $(\times 220)$. $(D)$ Semiquantification of these histological findings $($ mean \pm SE, $n=6)$. Statistical analysis: $* * P<0.01$ and $* * * P<0.001$ compared with the control group.

In early-stage CRD, nephrotic mice have been found to show tubular growth in the surviving nephrons, but this growth is no longer seen in the end stage, as a result of renal dysfunction (20). Therefore, we attempted initially to stimulate tubular growth to compensate for impaired renal function. Interestingly, tubular growth could be sustained at a significant level in the CRD mice treated with HGF. This indicates that reduction of the tubular parenchymal component, followed by subsequent interstitial expansion, was almost completely inhibited by the HGF injections. These histological changes are consistent with the prevention of increases in the BUN and sCr levels in the HGF-treated mice. The recovery from renal dysfunction after the HGF injections seems to be, at least in part, associated with compensatory tubular growth, which probably occurred in the remaining intact nephrons, while HGF may also activate tubular $\mathrm{Na}^{+}-\mathrm{K}^{+}$ATPase (37). Both of these effects are thought to lead to the restoration of renal dysfunction via the tubuloglomerular feedback system. It is also noteworthy that HGF has a unique morphogenic activity to induce branching tubulogenesis in renal epithelial cells (13), and that HGF is involved in morphogenesis and development of renal tissues $(13,38-40)$. Although it is quite difficult to obtain evidence that HGF exerts such a morphologic activity to accelerate histological as well as functional restoration in the kidney of ICGN mice, it is likely that HGF may also be involved in morphogenic processes (including activation of cellular functions), because growth-promoting activity of HGF alone is unlikely to be sufficient to support full restoration of renal tubules. Tubular cellular proliferation without concomitant expansion (or reconstruction) of normal architecture would result in the formation of nonfunctional renal tissue.

We found that HGF also attenuated the progression to glomerular obsolescence. As a possible explanation, the suppression of the tubulointerstitial and periglomerular myofibroblast formation by HGF may be associated with the attenuated glomerulosclerosis, because extraglomerular myofibroblasts accelerate further progression of glomerulosclerosis $(41,42)$. In brief, glomerular obsolescence occurs, in part, as a consequence of infiltration of glomerular tufts by interstitial myofibroblasts. If so, the attenuating effect of HGF on glomeruli may be attributable to the reduced myofibroblast formation in the interstitial areas. Recently, it was demonstrated that HGF is a potent endothelium-specific growth factor in vascular tissue and that a decrease in local production of HGF causes atherosclerosis (43). In view of the importance of a progressive loss of endothelial cells in the progression of glomerulosclerosis as well as of atherosclerosis, HGF may possibly attenuate progression of glomerulosclerosis through preventing endothelial injury.

So far, it has been suggested that severe and persistent glomerulosclerosis accelerates progression of tubular destruction in CRD, presumably through postglomerular vascular deterioration (5). To put it another way, the attenuation of glomerular injury by HGF may, at least in part, prevent further tubular injury and may facilitate the compensatory proliferation of intact tubular cells in response to exogenous HGF. Since it is generally difficult to determine whether glomerular injury causes or reflects tubulointerstitial fibrosis (3), we could 

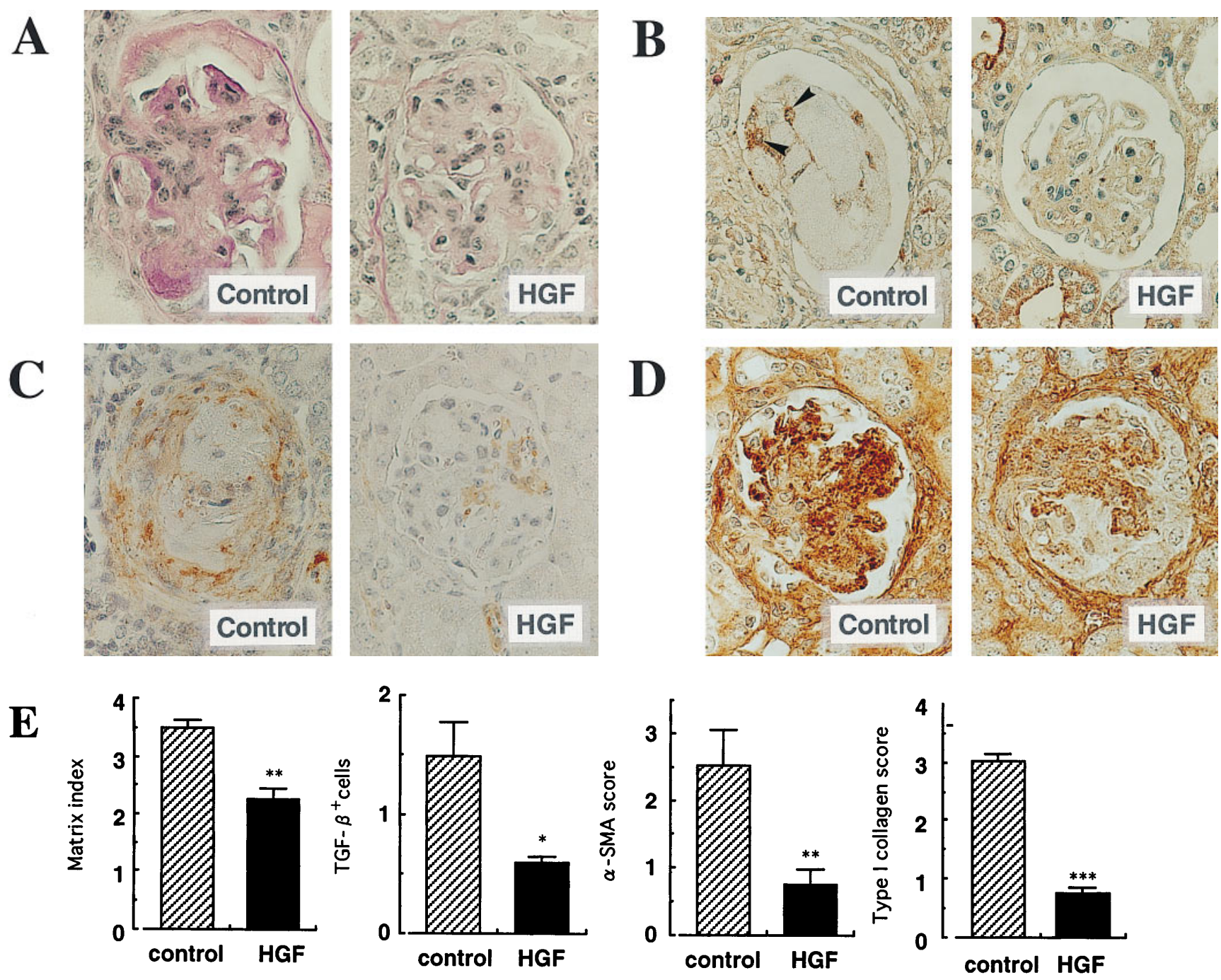

Figure 4. Attenuation of glomerular sclerosis and sclerogenetic events by HGF injections. $(A)$ Representative photomicrographs of sclerotic lesions $(\times 260$, PAS stain). $(B)$ Immunostaining for TGF- $\beta$ localized in intraglomerular cells (arrowheads, $\times 220)$. (C) Immunostaining for $\alpha$-SMA, a marker for periglomerular myofibroblast formation $(\times 220)$. $(D)$ Immunostaining for type I collagen in sclerotic glomeruli $(\times 220)$. $(E)$ Semiquantification of these histological findings (mean \pm SE, $n=6$ ). Statistical analysis: $* P<0.05, * * P<0.01$, and $* * * P<0.001$ compared with the control group.

not determine whether HGF primarily attenuated the tubular or glomerular injury. In our CRD model, however, the onset of the glomerular changes was obvious (from $3 \mathrm{wk}$ of age) before the onset of the tubular atrophy (from 14 wk of age) (20). Considering the age of the CRD mice subjected to HGF treatment (14 wk of age), we predict that the preventive effects of HGF may be exerted mainly through minimizing the tubular atrophy, leading to the suppression of interstitial myofibroblast formation and subsequent glomerular obsolescence.

Recent extensive studies have demonstrated that TGF- $\beta$ and PDGF are critically involved in the progression of tubulointerstitial expansion $(6,7,28)$ as well as of glomerulosclerosis $(29,44)$. In fact, suppression of expression of these fibrogenic growth factors could prevent glomerular injury $(45,46)$. Of interest, we discovered recently that the endogenous HGF concentration in renal tissue declined naturally in association with the development of tubular destruction, and that local
HGF levels correlated negatively with the intrarenal expressions of TGF- $\beta$ and ECM proteins in the CRD mouse kidney (data not shown). It is accepted widely that a phenotypic switch of mesangial or tubulointerstitial fibroblasts to myofibroblasts, a candidate(s) for ECM-producing cells, can be achieved by stimulation with TGF- $\beta$ or PDGF, and that myofibroblast formation is believed to be an important event in development of renal fibrosis $(28,41)$. We speculate that the decrease in local HGF expression either causes or reflects the increase in TGF- $\beta$ expression, eventually leading to myofibroblast accumulation and the manifestation of renal fibrosis. If so, an exogenous supplement of HGF would be effective for prevention of the relentless progression to end-stage renal failure. In fact, our study demonstrated that HGF injections led to reduced expressions of TGF- $\beta$ in the CRD model. In addition, renal expression of TGF- $\beta$ mRNA was markedly suppressed in the HGF-treated mice (data not shown). Downregulation of 

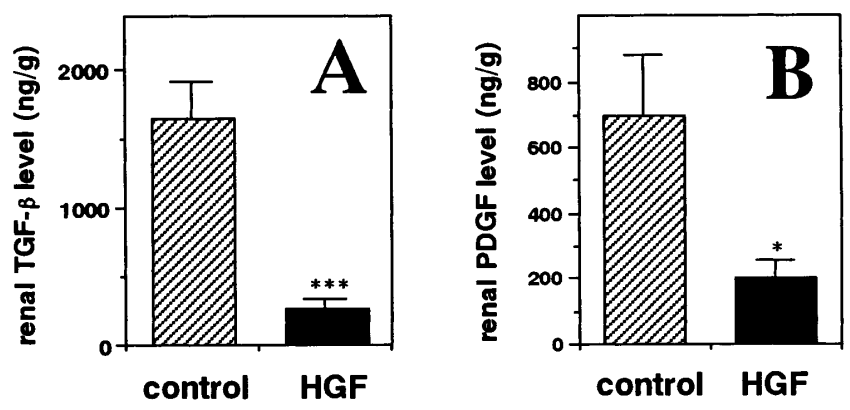

Figure 5. Decreases in renal concentrations of TGF- $\beta$ and PDGF proteins by HGF. $(A)$ Renal levels of TGF- $\beta$ protein. $(B)$ Renal levels of PDGF protein. Data are expressed as mean \pm SE $(n=6)$. Statistical analysis: $* P<0.05$ and $* * * P<0.001$ compared with the control group.

TGF- $\beta$ expression by HGF was also noted in rats with liver cirrhosis (34).

Recently, Takayama et al. reported that a transgenic mouse strain expressing HGF at extremely high levels manifests glomerulosclerosis and cystic tubules (47). This seems to conflict with our observations. In the transgenic mice, however, highly expressed HGF gene at local sites was, as they described, an important factor for the onset of the renal phenotype, because no correlation was seen between the renal lesions and serum HGF level (47). In contrast, no renal lesion was seen in other transgenic mice manifesting considerable HGF levels (48). We did not observe any lesions in various tissues, including the kidney, even through HGF was daily administrated into normal mice and rats at fully effective doses for 2 mo (data not shown). Taken together with our present results as well as with the previous results in other models for chronic organ injuries $(27,34-36)$, administration of HGF at pharmacologically effective doses seems to be safe, except when HGF is supplied at extremely high levels at local sites, including the kidney.

So far, there has been no clinically effective treatment for the pathological condition of CRD patients except through renal transplantation, but targeting strategy for fibrogenic molecules such as TGF- $\beta$ and PDGF $(45,46)$ would be available to directly prevent fibrosis. On the other hand, our strategy using supplement of HGF may also have a therapeutic potential in minimizing clinical manifestation of CRD, through reducing injured area by expanding intact area as well as through reducing renal expressions of TGF- $\beta$ and PDGF. Although further studies are needed to examine the effect of HGF on other CRD, we emphasize a possibility that HGF may be one of the promising candidates for preventing clinical manifestations of CRD. Additionally, our observations provide further insight into cellular and molecular pathogenesis of tubulointerstitial fibrosis, the best histological marker reflecting CRD.

\section{Acknowledgments}

We are grateful to $\mathrm{M}$. Ohara for critical reading of the manuscript and useful comments.

This work was supported by grants from the Japanese Ministry of Science, Culture and Education (07558243 to T. Kurosawa and 06283216 and 08408027 to T. Nakamura), and the Osaka Kidney Foundation (OKF 97-0003) to K. Matsumoto.

\section{References}

1. Lazarus, J.M., B.M. Denker, and W.F. Owen. 1996. Hemodialysis. In The Kidney. 5th Ed. B.M. Brenner, editor. W.B. Saunders, Philadelphia. 2424-2506.

2. Brenner, B.M., T.W. Meyer, and T.H. Hostetter. 1982. Dietary protein intake and the progressive nature of kidney disease: the role of hemodynamically mediated glomerular injury in the pathogenesis of progressive glomerular sclerosis in aging, renal ablation, and intrinsic renal disease. N. Engl. J. Med. 307:652-659.

3. Ong, A.C., and L.G. Fine. 1994. Loss of glomerular function and tubulointerstitial fibrosis: cause or effect? Kidney Int. 45:345-351.

4. Risdon, R.A., J.C. Sloper, and H.E. Wardener. 1968. Relationship between renal function and histological changes found in renal-biopsy specimens from patients with persistent glomerular nephritis. Lancet. 2:363-366.

5. Bohle, A., F. Strutz, and G.A. Muller. 1994. On the pathogenesis of chronic renal failure in primary glomerulopathies: a view from the interstitium. Exp. Nephrol. 2:205-210.

6. Eddy, A.A. 1996. Molecular insights into renal interstitial fibrosis. J. Am. Soc. Nephrol. 7:2495-2508.

7. Kliem, V., R.J. Johnson, C.E. Alpers, A. Yoshimura, W.G. Couser, K.M. Koch, and J. Floege. 1996. Mechanisms involved in the pathogenesis of tubulointerstitial fibrosis in 5/6-nephrectomized rats. Kidney Int. 49:666-678.

8. Lloyd, C.M., A.W. Minto, M.E. Dorf, A. Proudfoot, T.N.C. Wells, D.J. Salant, and J.C. Gutierrez-Ramos. 1997. RANTES and monocyte chemoattractant protein-1 (MCP-1) play an important role in the inflammatory phase of crescentic nephritis, but only MCP-1 is involved in crescent formation and interstitial fibrosis. J. Exp. Med. 185:1371-1380.

9. Hostetter, T.H. 1995. Progression of renal disease and renal hypertrophy. Anпu. Rev. Physiol. 57:263-278.

10. Nakamura, T., T. Nishizawa, M. Hagiya, T. Seki, M. Shimonishi, A. Sugimura, K. Tashiro, and S. Shimizu. 1989. Molecular cloning and expression of human hepatocyte growth factor. Nature. 342:440-443.

11. Miyazawa, K., H. Tsubouchi, D. Naka, K. Takahashi, M. Okigaki, N. Arakaki, H. Nakayama, S. Hirono, O. Sakiyama, K. Takahashi, et al. 1989. Molecular cloning and sequence analysis of cDNA for human hepatocyte growth factor. Biochem. Biophys. Res. Commun. 163:967-973.

12. Igawa, T., S. Kanda, H. Kanetake, Y. Saitoh, A. Ichihara, Y. Tomita, and T. Nakamura. 1991. Hepatocyte growth factor is a potent mitogen for cultured rabbit renal tubular epithelial cells. Biochem. Biophys. Res. Commun. 174:831-838.

13. Montesano, R., K. Matsumoto, T. Nakamura, and L. Orci. 1991. Identification of a fibroblast-derived epithelial morphogen as hepatocyte growth factor. Cell. 67:901-908.

14. Nagaike, M., S. Hirao, H. Tajima, S. Noji, S. Taniguchi, K. Matsumoto, and T. Nakamura. 1991. Renotropic functions of hepatocyte growth factor in renal regeneration after unilateral nephrectomy. J. Biol. Chem. 266:2278122784.

15. Joannidis, M., K. Spokes, T. Nakamura, D. Faletto, and L.G. Cantley. 1994. Regional expression of hepatocyte growth factor/c-met in experimental renal hypertrophy and hyperplasia. Am. J. Physiol. 267:F231-F236.

16. Kawaida, K., K. Matsumoto, H. Shimazu, and T. Nakamura. 1994. Hepatocyte growth factor prevents acute renal failure and accelerates renal regeneration in mice. Proc. Natl. Acad. Sci. USA. 91:4357-4361.

17. Miller, S.B., D.R. Martin, J. Kissane, and M.R. Hammerman. 1994. Hepatocyte growth factor accelerates recovery from acute ischemic renal injury in rats. Am. J. Physiol. 266:F129-F134.

18. Kurosawa, T., M. Okamoto, K. Yamada, and B.F. Yue. 1993. Nephrosis (nep): a new mouse mutation which causes albuminuria and other symptoms of nephrosis. Mouse Genome. 91:876-879.

19. Ogura, A., T. Asano, O. Suzuki, Y. Yamamoto, Y. Noguchi, H. Kawaguchi, and Y. Yamaguchi. 1994. Hereditary nephrotic syndrome with progression to renal failure in a mouse model (ICGN strain): clinical study. Nephron. 68:239-244.

20. Mizuno, S., B.F. Yue, M. Okamoto, Y. Horikawa, and T. Kurosawa. 1997. Diffuse glomerulosclerosis without tubular injury does not directly manifest renal dysfunction in nephrotic mice (ICGN strain). Exp. Nephrol. 5:498507

21. Shima, N., E. Tsuda, M. Goto, K. Yano, H. Hayasaka, M. Ueda, and K. Higashio. 1994. Hepatocyte growth factor and its variant with a deletion of five amino acids are distinguishable in their biological activity and tertiary structure. Biochem. Biophys. Res. Commun. 200:808-815.

22. Raij, L., S. Azar, and W. Keane. 1984. Mesangial immune injury, hypertension, and progressive glomerular damage in Dahl rats. Kidney Int. 26:137143.

23. Floege, J., R.J. Johnson, K. Gordon, H. Iida, P. Pritzl, A. Yoshimura, C. Campbell, C.E. Alpers, and W.G. Couser. 1991. Increased synthesis of extracellular matrix in mesangial proliferative nephritis. Kidney Int. 40:477-488.

24. Yo, Y., R. Morishita, J. Higaki, I. Kida, A. Moriguchi, M. Aoki, S. Kato, K. Matsumoto, T. Nakamura, and T. Ogihara. 1998. Function of hepatocyte growth factor as a local modulator in the kidney: potential role in pathogenesis of renal disease. Kidney Int. 53:50-58.

25. Laurens, W., C. Battaglia, C. Foglieni, R. Vos, B. Malanchini, B.V. 
Damme, Y. Vanrenterghem, G. Remuzzi, and A. Remuzzi. 1995. Direct podocyte damage in the single nephron leads to albuminuria in vivo. Kidney Int. 47:1078-1086.

26. Takehara, T., K. Matsumoto, and T. Nakamura. 1992. Cell densitydependent regulation of albumin synthesis and DNA synthesis in rat hepatocytes by hepatocyte growth factor. J. Biochem. 112:330-334.

27. Matsuda, Y., K. Matsumoto, T. Ichida, and T. Nakamura. 1995. Hepatocyte growth factor suppresses the onset of liver cirrhosis and abrogates lethal hepatic dysfunction in rats. J. Biochem. 118:643-649.

28. Tang, W.W., T.R. Ulich, D.L. Lacey, D.C. Hill, M. Qi, S.A. Kaufman, G.Y. Van, J.E. Tarpley, and J.S. Yee. 1996. Platelet-derived growth factor-BB induces renal tubulointerstitial myofibroblast formation and tubulointerstitial fibrosis. Am. J. Pathol. 148:1169-1180.

29. Border, W.A., and N.A. Noble. 1994. Transforming growth factor beta in tissue fibrosis. N. Engl. J. Med. 331:1286-1292.

30. Mizuno, S., Y. Mizuno-Horikawa, M. Okamoto, and T. Kurosawa. 1998. ACE inhibitor suppresses tubular expression of PDGF and attenuates progression of tubulo-interstitial fibrosis in a nephrotic mouse model. Nephron. In press.

31. Knecht, A., L.G. Fine, K.S. Kleinman, H.P. Rodemann, G.A. Muller, D.D. Woo, and J.T. Norman. 1991. Fibroblasts of rabbit kidney in culture. II. Paracrine stimulation of papillary fibroblasts by PDGF. Am. J. Physiol. 261: F292-F299.

32. Zarnegar, R., and G.K. Michalopoulos. 1995. The many faces of hepatocyte growth factor from hepatopoiesis to hematopoiesis. J. Cell Biol. 129:11771180 .

33. Ishiki, Y., H. Ohnishi, Y. Muto, K. Matsumoto, and T. Nakamura. 1992. Direct evidence that hepatocyte growth factor is a hepatotrophic factor for liver regeneration and has a potent antihepatitis effect in vivo. Hepatology. 16:12271235.

34. Yasuda, H., E. Imai, A. Shiota, N. Fujise, T. Morinaga, and K. Higashio. 1996. Antifibrogenic effect of a deletion variant of hepatocyte growth factor on liver fibrosis in rats. Hepatology. 24:636-642.

35. Matsuda, Y., K. Matsumoto, A. Yamada, T. Ichida, H. Asakura, Y. Komoriya, E. Nishiyama, and T. Nakamura. 1997. Preventive and therapeutic effects in rats of hepatocyte growth factor infusion on liver fibrosis/cirrhosis. Hepatology. 26:81-89.

36. Yaekashi, M., S. Nakamura, K. Ohnuma, T. Sasaki, T. Abe, K. Satoh, K. Matsumoto, T. Nakamura, T. Takahashi, and T. Nukiwa. 1997. Simultaneous or delayed administration of hepatocyte growth factor (HGF) equally repress the fibrotic changes in murine lung injury by bleomycin: a morphological study. Am. J. Respir. Clin. Care Med. 156:1937-1944.

37. Ishibashi, K., S. Sasaki, H. Sakamoto, Y. Nakamura, T. Hata, T. Naka- mura, and F. Marumo. 1992. Hepatocyte growth factor is a paracrine factor for renal epithelial cells: stimulation of DNA synthesis and $\mathrm{Na}, \mathrm{K}-\mathrm{ATPase}$ activity. Biochem. Biophys. Res. Commun. 182:960-965.

38. Barrow, E.J.G., O.F.T. Santos, K. Matsumoto, T. Nakamura, and S.K. Nigam. 1995. Differential tubulogenic and branching morphogenetic activities of growth factors: implications for epithelial tissue development. Proc. Natl. Acad. Sci. USA. 92:4412-4416.

39. Sonnenberg, E., D. Meyer, K.M. Weidner, and C. Birchmeier. 1993. Scatter factor/hepatocyte growth factor and its receptor, the c-met tyrosine kinase, can mediate a signal exchange between mesenchyme and epithelia during mouse development. J. Cell Biol. 123:223-235.

40. Woolf, A.S., M. Kolatsi-Joannou, P. Hardman, E. Andermarcher, C Moorby, L.G. Fine, P.S. Jat, M.D. Noble, and E. Gherardi. 1995. Role of hepatocyte growth factor/scatter factor and the Met receptor in the early development of the metanephron. J. Cell Biol. 128:171-184.

41. Nahas, A.M.E., K.E. Muchaneta, G. Zhang, A. Adam, and D. Goumenos. 1996. Phenotypic modulation of renal cells during experimental and clinical renal scarring. Kidney Int. 49(Suppl.):S23-S27.

42. Alpers, C.E., K.L. Hudkins, J. Floege, and R.J. Johnson. 1994. Human renal cortical cells with some features of smooth muscle cells participate in tubulointerstitial and crescentic glomerular injury. J. Am. Soc. Nephrol. 5:201-210.

43. Morishita, R., S. Nakamura, Y. Nakamura, M. Aoki, A. Moriguchi, I. Kida, Y. Yo, K. Matsumoto, T. Nakamura, J. Higaki, and T. Ogihara. 1997. Potential role of an endothelium-specific growth factor, hepatocyte growth factor, on endothelial damage in diabetes. Diabetes. 46:138-142.

44. Isaka, Y., Y. Fujiwara, N. Ueda, Y. Kaneda, T. Kamada, and E. Imai. 1993. Glomerulosclerosis induced by in vivo transfection of transforming growth factor-beta or platelet-derived growth factor gene into the rat kidney. $J$. Clin. Invest. 92:2597-2601.

45. Isaka, Y., D.K. Brees, K. Ikegaya, Y. Kaneda, E. Imai, N.A. Noble, and W.A. Border. 1996. Gene therapy by skeletal muscle expression of decorin prevents fibrotic disease in rat kidney. Nature Med. 2:418-423.

46. Johnson, R.J., E.W. Raines, J. Floege, A. Yoshimura, P. Pritzl, C. Alpers, and R. Ross. 1992. Inhibition of mesangial cell proliferation and matrix expansion in glomerulonephritis in the rat by antibody to platelet-derived growth factor. J. Exp. Med. 175:1413-1416.

47. Takayama, H., W.J. LaRochelle, S.G. Sabnis, T. Otsuka, and G. Merlino. 1997. Renal tubular hyperplasia, polycystic disease, and glomerulosclerosis in transgenic mice overexpressing hepatocyte growth factor/scatter factor. Lab. Invest. 77:131-138.

48. Shiota, G., T.C. Wang, T. Nakamura, and E.V. Schmidt. 1994. Hepatocyte growth factor in transgenic mice: effects on hepatocyte growth factor, liver generation and gene expression. Hepatology. 19:962-972. 